\title{
The effects of simvastatin on hippocampal caspase-3 and Bcl-2 expression following kainate-induced seizures in rats
}

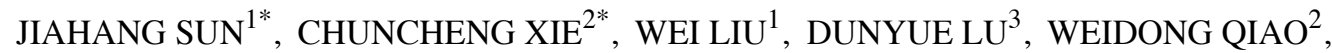 \\ QI HUANG ${ }^{4}$, ZHIHUI HUO ${ }^{2}$, HONG SHEN ${ }^{2}$ and ZHIGUO LIN ${ }^{2}$ \\ ${ }^{1}$ Department of Neurosurgery, The Second Affiliated Hospital of Harbin Medical University, Harbin; ${ }^{2}$ Department \\ of Neurosurgery, The First Affiliated Hospital of Harbin Medical University, Harbin, P.R. China; ${ }^{3}$ Department \\ of Psychiatry, State University of New York, Downstate Medical Center, Brooklyn, NY, USA; \\ ${ }^{4}$ Department of Anatomy, Harbin Medical University, Harbin, P.R. China
}

Received March 14, 2012; Accepted May 4, 2012

DOI: $10.3892 /$ ijmm.2012.1076

\begin{abstract}
Status epilepticus (SE) causes neuronal loss and apoptosis by inducing several apoptosis-regulatory genes. Two such genes, cysteinyl aspartate-specific protease-3 (caspase-3), an apoptosis activator, and B-cell leukemia-2 (Bcl-2), an apoptosis suppressor, are tightly regulated for their expression and activation. Statins, inhibitors of HMG-CoA reductase, have been recently recognized as neuroprotective drugs. However, their underlying mechanisms are still unclear. In this study, we examined the neuroprotective effects of simvastatin in a rat model of SE induced by kainic acid (KA). Feeding of simvastatin for 3 days after kainate injection rescued SE-induced neuronal apoptosis, as determined by histological examination of brain sections at the level of the dorsal hippocampus. Semi-quantitative RT-PCR showed that SE treatment markedly increased caspase-3 mRNA expression and reduced Bcl-2 mRNA expression in the hippocampus. Similarly, western blot analysis and immunohistochemical analysis of the rat hippocampus demonstrated that under SE treatment, caspase-3 protein levels significantly increased and peaked at $72 \mathrm{~h}$, whereas Bcl-2 protein levels decreased from 6-24 h following SE. Interestingly, simvastatin could reverse the aforementioned SE-induced changes, suggesting that the neuroprotective effects of simvastatin against neuronal apoptosis may be achieved by inhibiting caspase- 3 expression and increasing Bcl-2 expression.
\end{abstract}

\section{Introduction}

Status epilepticus (SE) in adult rodents and humans can cause hippocampal neuronal loss and may result in temporal lobe

Correspondence to: Dr Zhiguo Lin, Department of Neurosurgery, The First Affiliated Hospital of Harbin Medical University, Harbin 150000, P.R. China

E-mail: linzhiguo@hotmail.com

*Contributed equally

Key words: simvastatin, status epilepticus, B-cell leukemia-2, cysteinyl aspartate-specific protease-3, kainic acid, hippocampus epilepsy (TLE) (1-3). Neuronal loss during chronic epilepsy mainly results from cell apoptosis or necrosis (4-6), which is triggered by the activation of a number of signal transduction factors (7). Kainic acid (KA), an analogue of the excitatory amino acid glutamate, has been widely used in inducing TLE in animal models $(8,9)$. KA-induced SE in the amygdaloid complex activates several signal transduction factors, including B-cell leukemia-2 (Bcl-2), Bcl-2-associated X protein (Bax), and cysteinyl aspartate-specific protease-3 (caspase-3) $(10,11)$. In the KA model, deletion or inhibition of pro-apoptotic genes protects the brain against seizure-induced neuronal death $(12,13)$. Since currently available anti-epileptic drugs merely treat symptoms but do not cure the disease, it is imperative to develop neuroprotective drugs that prevent apoptosis after SE.

Statins, inhibitors of HMG-CoA reductase, inhibit cellular synthesis of cholesterol and isoprenoids and are commonly used to reduce cholesterol levels in humans. In addition to their lipid-lowering and thus beneficial cardiovascular effects, statins have also been suggested to exert neuroprotective actions in the central nervous system. For example, statins play antiinflammatory and vasoprotective roles in cultured brain cells and endothelial cells (14). Statins inhibit a number of inflammatory processes important to brain damage and suppress the secretion of cytokines during spinal cord injury and ischemic stroke $(15,16)$. The neuroprotective effects of statins have also been reported in various diseases, including traumatic brain injury, brain ischemia, and Alzheimer's disease, in both animal models and clinical studies (17-20). In KA-induced seizure, treatment with statins provides anti-apoptotic effects (21). However, the mechanisms underlying the anti-apoptotic role are not clear. In this study, we examined whether simvastatin regulates apoptosis and exerts its neuroprotective effects by modulating the expression of Bcl-2 and caspase- 3 .

\section{Materials and methods}

Ethics statement. Animal care and handling was conducted in compliance with the Chinese Animal Welfare Act and was approved by the Medical Ethics Committee of the First Clinical College of Harbin Medical University (Approval ID, 201001). 
Animals. Adult male Wistar rats ( $\mathrm{n}=120)$, weighing 180-200 g, were provided by the Animal Center of Jilin University, China. The rats were housed in individual cages in a controlled environment (constant $22-25^{\circ} \mathrm{C} ; 50-60 \%$ humidity; $12 / 12 \mathrm{~h}$ light/ dark cycle, lights on at 7 a.m.) for at least 1 week before being used in the experiment. The rats had free access to standard laboratory food and water. In addition, all efforts were made to minimize animal suffering and to use only the number of animals necessary to produce reliable scientific data. All the experiments were conducted in the morning to avoid circadian variations.

Experimental groups and drug administration. The rats were randomly divided into 4 groups ( $n=30$ per group): a saline group (sham), an epilepsy group, an epilepsy plus saline group, and an epilepsy plus simvastatin group. In the sham group, rats received saline intraperitoneally. In the epilepsy group, KA was dissolved in isotonic saline $(\mathrm{pH} 7.3)$ and administered intraperitoneally to rats at a dose of $10 \mathrm{mg} / \mathrm{kg}$. In the epilepsy plus saline group, SE were induced in rats with KA injection followed by oral administration of saline starting at $0.5 \mathrm{~h}$ after SE once a day for 3 consecutive days. In the epilepsy plus simvastatin group, rats were subjected to KA lesions followed by oral administration of simvastatin $\left(1 \mathrm{mg} / \mathrm{kg} /\right.$ day) (Zocor $^{\circledR}$; MSD, USA), starting at $0.5 \mathrm{~h}$ after SE for 3 consecutive days. The dose was selected according to our previous study $(22,23)$. The rats were sacrificed at indicated time points after SE or sham operation.

$K A$-induced rat seizure model. SE was induced in rats using KA administration. After KA was administered, the behavior of rats was observed for 3-4 h and documented to determine the duration and severity of seizure activity using a previously established seizure scoring scale (24). This method was widely used to study epilepsy in rodents. The behavior of rats was divided into 5 stages: stage 1, immobility; stage 2 , forelimb and/or tail extension; stage 3 , head bombing, as well as forelimb clonus with rearing and falling; stage 4, minor clonic seizures; stage 5, severe tonic-clonic seizures; and stage 6 , death. Only those rats exhibiting at least $2 \mathrm{~h}$ of continuous stage 4/5 seizures were included in this study. Seizure parameters monitored included latency of convulsions and duration of severe (stage 4/5) seizure activity.

Histological analysis. Neuronal damage was assessed by histological examination of brain sections from the dorsal hippocampus of rats sacrificed at $6,12,24,48,72$ or $96 \mathrm{~h}$ after SE or sham operation. The rats were deeply anesthetized with $4 \%$ halothane and then transcardially perfused with $200 \mathrm{ml}$ of heparinized $0.9 \%$ saline followed by $500 \mathrm{ml}$ of $4 \%$ paraformaldehyde (no. 158127; Sigma-Aldrich, Beijing, China) in $0.1 \mathrm{~mol} / 1$ of phosphate-buffered saline ( $\mathrm{pH} 7.4)$ (P5368; Sigma-Aldrich). After rats were decapitated, the brains were immersed in $4 \%$ paraformaldehyde for 3 days, embedded in paraffin, and sliced on a rotary microtome into 6-mm thick sections. Coronal sections consisting of the dorsal hippocampus were selected and processed for hematoxylin and eosin (H\&E) staining. The paraffin-embedded sections were dewaxed by baking at $55-65^{\circ} \mathrm{C}$ for $45-60 \mathrm{~min}$, cleaned with xylene three times for $5 \mathrm{~min}$ each, and rehydrated with $100 \%$ ethanol twice for $5 \mathrm{~min}$ and $70 \%$ ethanol once for $5 \mathrm{~min}$. After 5 -min washes in distilled water, the sections were stained with hematoxylin (H9627; Sigma-Aldrich) for 2 min and then washed twice in running water for $1 \mathrm{~min}$. The sections were dipped in ammonium hydroxide solution and then washed again using running water for $1 \mathrm{~min}$. After being rinsed in graded ethanol (80\% ethanol for $1 \mathrm{~min}$ and $95 \%$ ethanol for $1 \mathrm{~min}$ ), each section was dipped 10 times in eosin-Y (E4009; Sigma-Aldrich) for 30-45 sec for counterstaining. Following two additional rinses in $100 \%$ ethanol for $1 \mathrm{~min}$ each, the sections were dehydrated in a graded series of alcohol, cleared in xylene, and coverslipped with Permount.

Semi-quantitative RT-PCR. The rats were anesthetized with $4 \%$ halothane and decapitated at 12 and $72 \mathrm{~h}$ after $2 \mathrm{~h}$ of SE. The hippocampus was then immediately isolated and put on an icecold glass stage. Total-RNA was extracted from the hippocampus using an RNA isolation reagent, TRIzol (TRIzol ${ }^{\circledR}$ Reagent; Invitrogen Life Technologies, Beijing, China), and reverse transcription was performed using oligo(dt) priming according to the manufacturer's instructions (ThermoScript ${ }^{\mathrm{TM}}$ RT-PCR System; Invitrogen Life Technologies). Primer sequences were as follows: rat caspase-3 (GenBank accession no. NM_012922), F, 5'-CTGGACTGCGGTATTGAG-3' and R, 5'-GGAACATCGGATTTGATT-3'; rat Bcl-2 (GenBank accession no. NM_021850), F, 5'-CTACCCAAGTTAGCATT CC-3' and R, 5'-CAAAGTCCCTATTTATCCCT-3'; and rat $\beta$-actin (GenBank accession no. NM_031144), F, 5'-AGCCA TGTACGTAGCCATCC-3' and R, 5'-GCTGTGGTGGTG AAGCTGTA-3'. The PCR reactions were conducted as follows: 5 min at $94^{\circ} \mathrm{C} ; 35$ cycles (for caspase- 3 and Bcl-2) or 30 cycles (for $\beta$-actin) of $30 \mathrm{sec}$ at $94^{\circ} \mathrm{C}, 30 \mathrm{sec}$ at $55^{\circ} \mathrm{C}$, and $30 \mathrm{sec}$ at $72^{\circ} \mathrm{C}$; and final elongation for $10 \mathrm{~min}$ at $72^{\circ} \mathrm{C}$. The amplified DNA fragments were 465 bp for caspase-3, 389 bp for Bcl-2, and $222 \mathrm{bp}$ for $\beta$-actin. The PCR products were run on a $2 \%$ agarose gel and visualized by UV light. Band densities were quantified using the Scion Image software (Scion Corporation Frederick, MD, USA) and signals from caspase-3 and Bcl-2 were normalized to those from the housekeeping gene $\beta$-actin.

Western blot analysis. Rats were sacrificed at 6, 12, 24, 48, 72 or $96 \mathrm{~h}$ after $2 \mathrm{~h}$ of SE or sham operation. Immediately after decapitation, the hippocampus was quickly dissected and then homogenized using a Dounce homogenizer and lysed on ice in $400 \mu \mathrm{l}$ of RIPA buffer [50 mM Tris- $\mathrm{HCl}$ ( $\mathrm{pH} 7.4$ ), $150 \mathrm{mM} \mathrm{NaCl}, 1 \mathrm{mM}$ PMSF, $1 \mathrm{mM}$ EDTA, $1 \%$ Triton X-100, $0.5 \%$ sodium deoxycholate, and $0.1 \%$ SDS]. Proteins, $20 \mu \mathrm{g}$ per lane as determined using the BCA protein assay kit (no. 23227; Pierce, Rockford, IL, USA), were separated on $12 \%$ SDS-polyacrylamide gels and transferred to polyvinylidene difluoride membranes (RPN2020F; GE Healthcare, Beijing, China). The membranes were first probed with primary antibody against cleaved caspase-3 (1:1,000; no. 9664; Cell Signaling Technology, Inc., Danvers, MA, USA; recognizing the active form p17 of caspase-3), Bcl-2 (1:50; ab7973; Abcam Inc., Cambridge, MA, USA), or $\beta$-actin (1:200; sc-47778; Santa Cruz Biotechnology, Inc., Santa Cruz, CA, USA). The specificity of the immunoreactivity for each antibody was confirmed by preabsorption experiments. After washes, the membranes were then incubated with horseradish peroxidase- 
conjugated secondary antibodies (1:5,000; for caspase-3 and Bcl-2: sc-2004; and for $\beta$-actin: sc-2005; were from Santa Cruz Biotechnology, Inc.). Subsequently, the protein bands were visualized with ECL (RPN2109; GE Healthcare) and quantified by densitometry.

Immunohistochemical staining. Rats were anesthetized and perfused as described for the histological analysis. Coronal sections including the dorsal hippocampus were selected and processed for immunohistochemical staining. The paraffin sections were baked for $2 \mathrm{~h}$ in an oven, deparaffinized in xylene, and rehydrated in graded ethanol solutions. After three 5-min washes in $0.01 \mathrm{M}$ PBS (pH 7.4), the sections were microwaved in $0.01 \mathrm{M}$ sodium citrate buffer ( $\mathrm{pH}$ 6.0) for $10 \mathrm{~min}$, cooled to room temperature naturally, and then washed three times in PBS, 5 min each. The sections were incubated in $3 \%$ hydrogen peroxide for $10 \mathrm{~min}$, washed using the same procedure as above, and then blocked in 10\% normal goat serum for $1 \mathrm{~h}$ at room temperature. Rabbit monoclonal antibodies against cleaved caspase-3 or Bcl-2 (1:200; no. 2870; Cell Signaling Technology, Inc.) were diluted in the recommended antibody diluent (no. 8112; Cell Signaling Technology, Inc.) and incubated with sections overnight at $4^{\circ} \mathrm{C}$. After three rinses in PBS, the sections were incubated with biotinylated goat anti-rabbit secondary antibody $(1: 2,000)$ for $1 \mathrm{~h}$ at room temperature and then with avidin-biotin-peroxidase solution (ABC reagent) for $30 \mathrm{~min}$ at room temperature. Following three additional washes in PBS, the sections were treated with 3,3'-diaminobenzidine tetrahydrochloride (DAB) for $2 \mathrm{~min}$, counterstained in hematoxylin, dehydrated, and coverslipped. Normal goat serum, biotinylated goat anti-rabbit secondary antibody, $\mathrm{ABC}$ reagent, and $\mathrm{DAB}$ were all in the $\mathrm{ABC}$ staining kit (sc-2018; Santa Cruz Biotechnology, Inc.). To assess nonspecific immunostaining in our study, control samples were stained only with secondary antibody, and no labeling was detected. To count the number of positive neurons, 10 microscopic fields in the hippocampus, at a magnification of $50 \mu \mathrm{m}$, were randomly chosen from each section, and the caspase-3- and Bcl-2-positive neurons were quantified using the Image-Pro Plus software (Media Cybernetics, Inc., Shanghai, China).

Statistical analysis. Data were expressed as mean \pm standard deviation and analyzed using one-way analysis of variance (ANOVA) and post hoc Fisher's PLSD after normality of the distribution was proved. $\mathrm{P}<0.05$ was considered statistically significant.

\section{Results}

Simvastatin rescues $S E$-induced neuronal apoptosis in the hippocampal CAl region. To examine the effects of SE on the hippocampal CA1 neurons and the role of simvastatin in this process, we conducted H\&E staining of the hippocampal CA1 region of rats. We demonstrated that 2-h SE induced selective neuronal death. Compared with hippocampal CA1 sections from the sham group rats (Fig. 1A and B), those from the epilepsy group showed partial cell death and neuronal loss following the 2-h SE. Typical morphological characteristics of apoptosis were observed in CA1 neurons, including cell shrinkage, nuclear condensation, and fragmentation

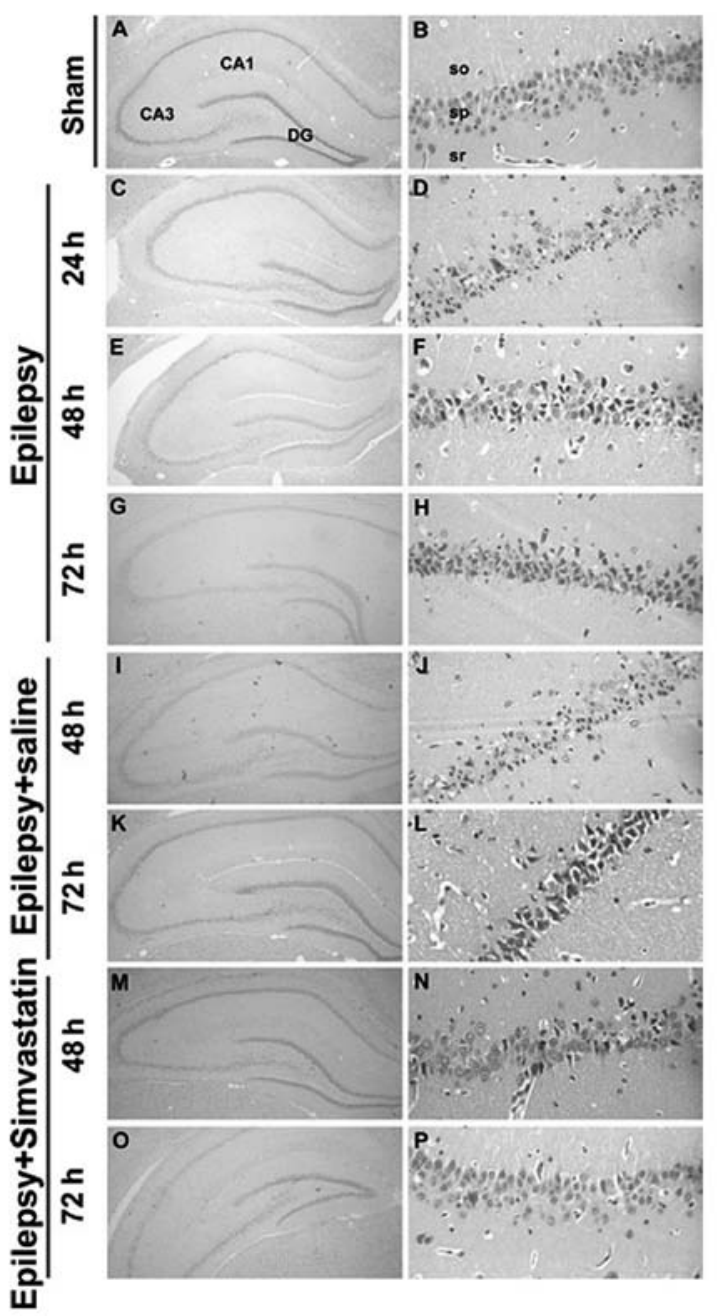

Figure 1. Simvastatin rescues SE-induced neuronal apoptosis in the hippocampal CA1 region. Neuronal damage and apoptosis were assessed by $\mathrm{H} \& \mathrm{E}$ staining and histological examination of brain sections from the hippocampal CA1 region. (A and $\mathrm{B}$ ) In the sham group, brain sections revealed normal neurons in the hippocampus. $(\mathrm{C}-\mathrm{H})$ In the epilepsy group, sections from 24-72 $\mathrm{h}$ after a 2-h SE showed neuronal loss and apoptosis, displaying cell shrinkage, nuclear condensation, and fragmentation. At $72 \mathrm{~h}$, a dramatic loss of neurons was observed, and the number of apoptotic neurons increased significantly. (I-L) The epilepsy plus saline group showed similar levels of neuronal loss and apoptosis as the epilepsy group. (M-P) Simvastatin treatment, as shown by 48- and 72-h post-SE sections, protected neurons from SE-induced neuronal loss and apoptosis.

(Fig. 1C-H). Twenty-four hours after SE, the CA1 cell layer showed a dramatic loss of neurons (Fig. 1C and D). The number of apoptotic neurons kept increasing at 48 and $72 \mathrm{~h}$ (Fig. 1E-H). Saline intake did not diminish the effect on neuronal death induced by SE (Fig. 1I-L). In contrast, simvastatin administration markedly rescued SE-induced neuronal apoptosis, especially at 48 and $72 \mathrm{~h}$ (Fig. 1M-P).

Simvastatin reverses SE-induced changes in caspase-3 and Bcl-2 mRNA expression in the hippocampus. To analyze the potential mechanisms underlying the neuroprotective role of simvastatin, we examined the mRNA expression of the proapoptotic gene, caspase-3, and the anti-apoptotic gene, Bcl-2, 72 and $12 \mathrm{~h}$ following SE, respectively. The semiquantitative RT-PCR results showed that the basal expression levels of caspase- 3 and Bcl-2 mRNA were readily detectable in the 

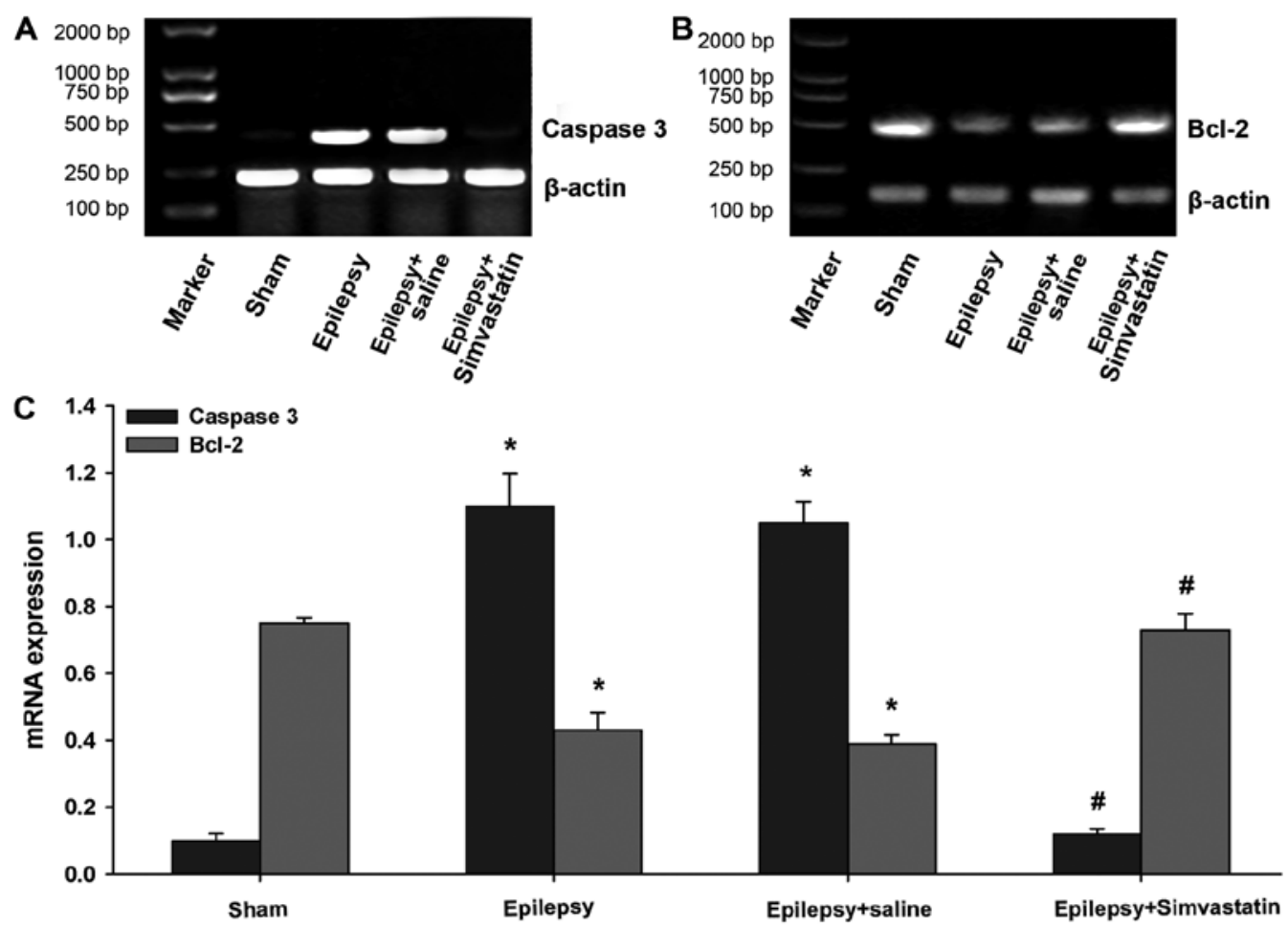

Figure 2. Simvastatin reverses SE-induced changes in caspase-3 and Bcl-2 mRNA expression in the hippocampus. The mRNA expression of the pro-apoptotic gene, caspase-3, and the anti-apoptotic gene, Bcl-2, in the hippocampus was examined 72 and $12 \mathrm{~h}$ after SE, respectively. The mRNA levels were measured using semiquantitative RT-PCR ( $\mathrm{n}=6$ per group). (A) SE significantly increased caspase-3 mRNA expression, and this increase was abolished by intake of simvastatin $(10 \mathrm{mg} / \mathrm{kg}$ ), but not saline. (B) SE significantly decreased Bcl-2 mRNA expression, and this reduction was rescued by simvatastin intake (1 mg/kg), but not saline. (C) Quantification of caspase-3 and Bcl-2 mRNA levels. Expression of target genes was normalized to that of $\beta$-actin. ${ }^{*} \mathrm{P}<0.01 \mathrm{vs}$. sham; ${ }^{*} \mathrm{P}<0.01 \mathrm{vs}$. epilepsy.

hippocampus of the sham rats (Fig. 2). SE significantly increased the caspase- 3 mRNA expression $72 \mathrm{~h}$ post-SE (Fig. 2A and C) $(\mathrm{P}<0.01)$ and significantly decreased Bcl-2 mRNA expression $12 \mathrm{~h}$ post-SE (Fig. 2B and $\mathrm{C})(\mathrm{P}<0.01)$. Saline intake did not affect the mRNA expression of caspase-3 and Bcl-2 following $\mathrm{SE}$ (Fig. 2) ( $\mathrm{P}<0.01$ vs. the sham group). Interestingly, simvastatin treatment completely reversed SE-induced changes in both caspase- 3 and Bcl-2 mRNA expression, to levels not significantly different from that of the sham group (Fig. 2) ( $\mathrm{P}<0.01$ vs. the epilepsy group; $\mathrm{P}>0.05$ vs. the sham group).

Simvastatin reverses SE-induced changes in caspase-3 and Bcl-2 protein expression in the hippocampus (western blot analysis). We further confirmed the influence on caspase-3 and Bcl-2 expression by examining protein levels. After SE-induced activation, full length caspase-3 (32 kDa) is cleaved into 2 mature subunits, p17 (17 kDa) and p12 (12 kDa). In this study, using an antibody specific to $\mathrm{p} 17$, we compared the levels of activated caspase- 3 in the 4 groups. As expected, p17 staining was not detected in the sham hippocampus, whereas SE markedly increased the expression of activated caspase- 3 . This increase began at $24 \mathrm{~h}$ and peaked at $72 \mathrm{~h}$ after $\mathrm{SE}$ (Fig. $3 \mathrm{~A}$ and $\mathrm{C})(\mathrm{P}<0.05$ vs. the sham group). Treatment with simvastatin, but not saline, significantly reduced the SE-induced increase of activated caspase-3 levels (Fig. 3A and $\mathrm{C})(\mathrm{P}<0.05$ vs. the epilepsy group).

Despite comparable Bcl-2 protein expression in the sham and epilepsy groups immediately after the 2-h SE (i.e., $0 \mathrm{~h}$ ), Bcl-2 levels were significantly decreased from 6 to $24 \mathrm{~h}$ (Fig. 3B and $\mathrm{D})(\mathrm{P}<0.05$ vs. the sham group). Intake of simv- astatin, but not saline, significantly reversed the reduction in Bcl-2 protein levels following SE (Fig. 3B and D) $(\mathrm{P}<0.05$ vs. the epilepsy group).

Simvastatin reverses $S E$-induced changes in caspase-3 and Bcl-2 protein expression in the hippocampus (immunohistochemical analysis). To characterize the cellular distribution of caspase-3 and Bcl-2 activation after SE, we performed immunohistochemical staining of caspase- 3 in brain sections $72 \mathrm{~h}$ after SE and of Bcl-2 in sections $12 \mathrm{~h}$ after SE. Neurons in the hippocampal CA1 region prepared from sham animals showed some distribution of caspase-3 (Fig. 4A) and Bcl-2 (Fig. 5A) staining in the nucleus or cytoplasm. Under SE treatment, the caspase- 3 expression in the hippocampal CA1 pyramidal cell layer increased significantly at 72 h (Fig. 4B). In contrast, $\mathrm{SE}$ treatment significantly decreased the expression of $\mathrm{Bcl}-2$, which was predominantly localized in the cytosol (Fig. 5B). Saline intake did not affect SE-induced expression patterns of caspase-3 (Fig. 4C) or Bcl-2 (Fig. 5C). In contrast, simvastatin treatment significantly reduced $\mathrm{SE}$-induced caspase- 3 activation (Fig. 4D) and, rescued SE-induced reduction in Bcl-2 expression (Fig. 5D) in the hippocampal CA1 region.

To quantitatively analyze caspase- 3 and Bcl-2 expression in the hippocampus, the mean intensity was analyzed by counting the number of positive neurons. Under SE treatment, the number of caspase-3-positive neurons increased significantly at $72 \mathrm{~h}$ (Fig. 4E) ( $\mathrm{P}<0.01$ vs. the sham group), whereas the number of Bcl-2-positive neurons decreased significantly at $12 \mathrm{~h}$ (Fig. 5E) $(\mathrm{P}<0.05$ vs. the sham group). Treatment with simvastatin, but not saline, could reverse SE-induced changes 
A

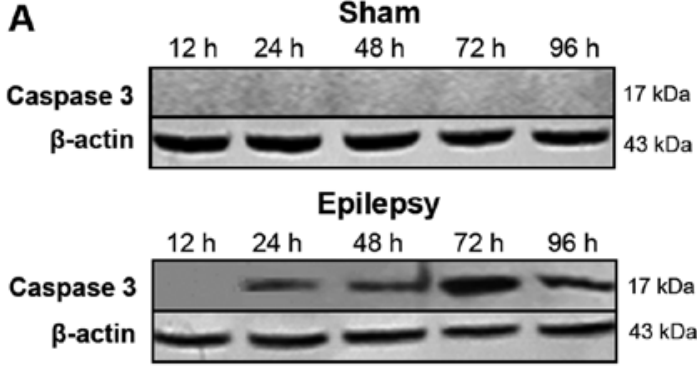

B

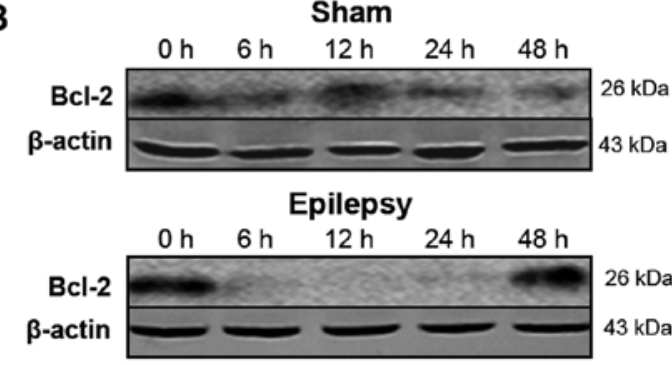

C

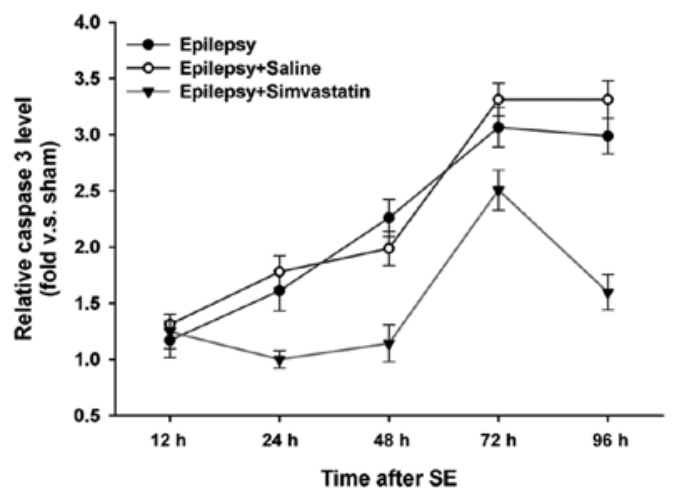

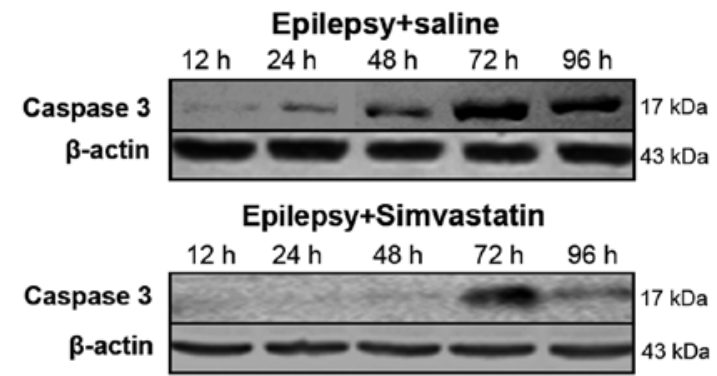

Epilepsy+saline

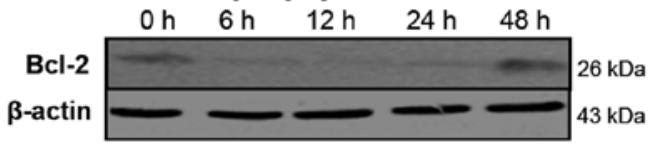

Epilepsy+Simvastatin

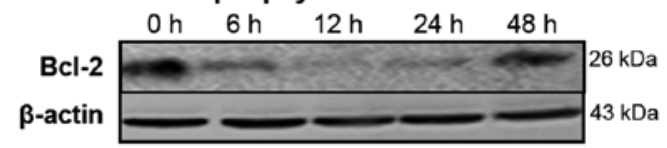

D

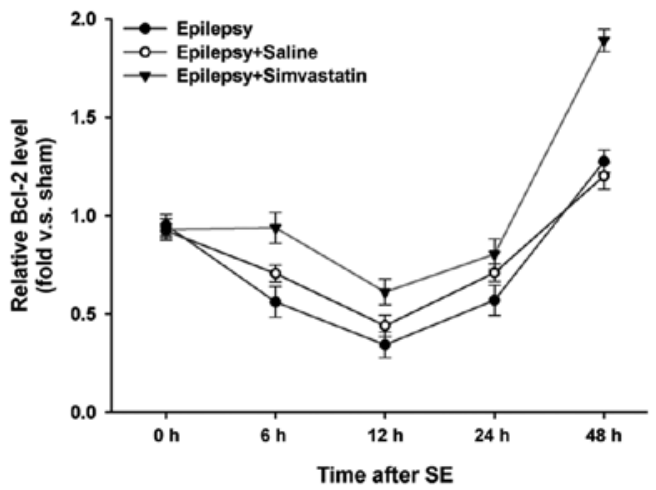

Figure 3. Simvastatin reverses SE-induced changes in caspase-3 and Bcl-2 protein expression in the hippocampus (western blot analysis). Western blot analyses showing representative images of caspase-3 and Bcl-2 protein expression in the hippocampus from the sham, epilepsy, epilepsy plus saline, and epilepsy plus simvastatin groups (the sham group, $\mathrm{n}=3$; each time point in the other three groups, $\mathrm{n}=3$ ). Band signals of target genes were normalized to those of $\beta$-actin. (A) The expression of the large cleavage form ( $17 \mathrm{kDa})$ of caspase-3 increased from $24 \mathrm{~h}$ after SE and peaked at $72 \mathrm{~h}$. Under simvastatin $(1 \mathrm{mg} / \mathrm{kg})$ treatment, the level of caspase-3 after SE decreased and returned to the basal level at $96 \mathrm{~h}$. (B) Bcl-2 protein levels decreased after SE from 6-24 h. Treatment with simvastatin $(1 \mathrm{mg} / \mathrm{kg}$ ) after SE upregulated the level of Bcl-2 expression. (C and D) Quantification of caspase-3 and Bcl-2 protein levels, showing fold changes vs. the sham controls. ${ }^{*} \mathrm{P}<0.05$ vs. sham; ${ }^{\#} \mathrm{P}<0.05$ vs. epilepsy.

in the number of caspase-3- and Bcl-2-positive neurons (Fig. 4E) ( $\mathrm{P}<0.01$ vs. the epilepsy group) (Fig. 5E) ( $\mathrm{P}<0.05$ vs. the epilepsy group).

\section{Discussion}

SE as well as brief and/or repetitive seizures associated with epilepsy cause neuronal loss in the brain (25). In the KA-induced SE rat model, pyramidal cells in the CA1 and CA3 regions of the hippocampus undergo apoptosis and death $(26,27)$. This apoptotic process in degenerating neurons is characterized by morphological changes, altered expression of Bcl-2-family proteins, and activation of the caspase family of cell-death proteases (28-30). Consistent with previous reports, in this study, we observed that under SE treatment, from 12 to $48 \mathrm{~h}$, a small number of neurons demonstrate the typical characteristics of apoptosis, including cell shrinkage, nuclear condensation, and fragmentation, and the number of apoptotic neurons increases significantly at $72 \mathrm{~h}$.

Apoptosis is triggered by a series of caspase cascades $(31,32)$. Caspase- 3 , a crucial apoptotic regulator that is acti- vated by caspases- 9 or -8 , is activated during seizure-induced neuronal death $(32,33)$. Controversially, other studies have suggested that caspase- 3 does not contribute significantly to SE-induced neuronal necrosis, because caspase- 3 activation was not detected 6 and $24 \mathrm{~h}$ after 2 -h SE $(34,35)$. In our experiment, caspase- $3 \mathrm{mRNA}$ and protein expression increased in hippocampal neurons $24 \mathrm{~h}$ after the 2-h SE and peaked at $72 \mathrm{~h}$. Our results strongly support the theory that caspase- 3 plays an important role in neuronal cell death during SE. The sham control rats express only low basal levels of caspase-3 mRNA and do not exhibit activated caspase-3 protein, and this low level of expression may be interpreted as mediating normal brain development.

$\mathrm{Bcl}-2$, the founding member of the Bcl-2 family, suppresses apoptosis primarily via effects on mitochondria and one of its mechanisms is preventing cytochrome $\mathrm{c}$ release $(36,37)$. Under KA-induced SE, Bcl-2 family members, including $\mathrm{Bax}$ and $\mathrm{Bcl}-2$, are activated in the amygdaloid complex $(36,37)$. In the present study, Bcl-2 mRNA and protein expression decreased $6 \mathrm{~h}$ after the 2-h SE and reached the trough level at $12 \mathrm{~h}$, suggesting that SE may accelerate 


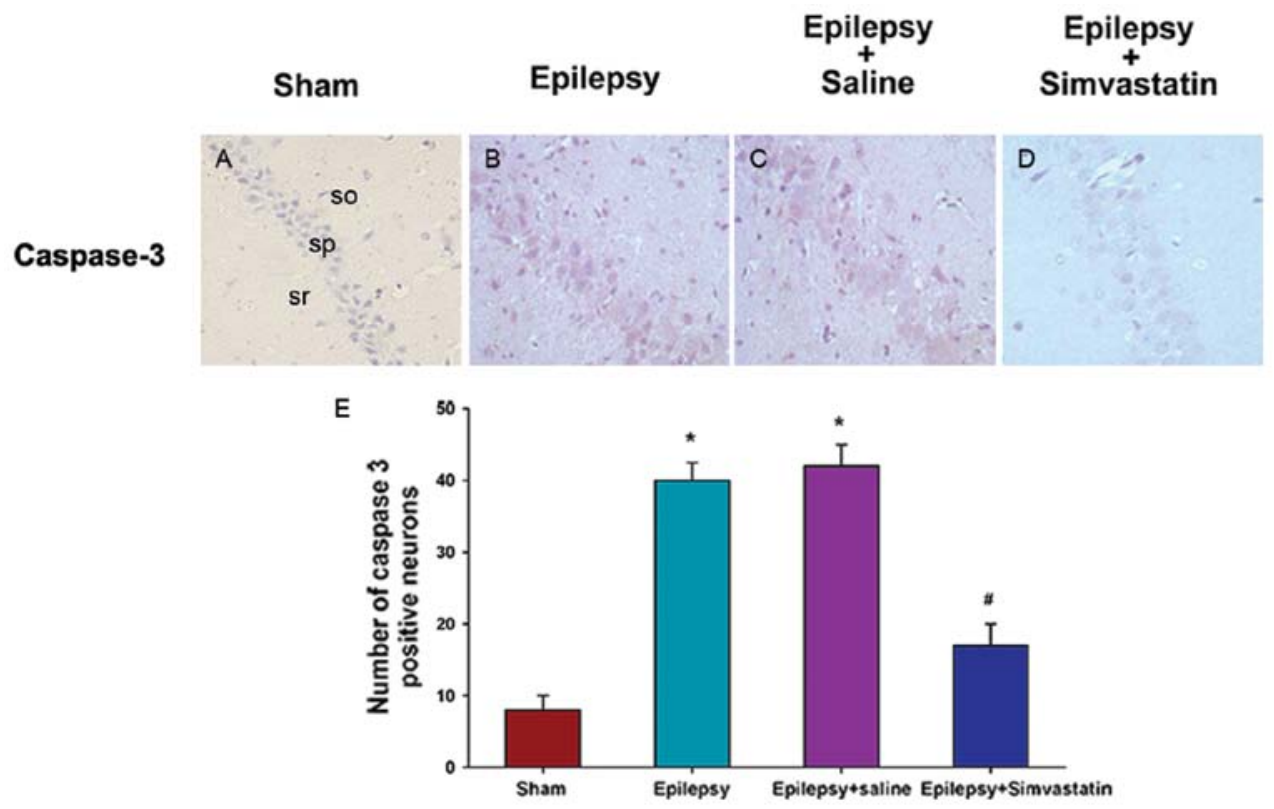

Figure 4. Simvastatin abolishes SE-induced increase in caspase-3 protein expression in the hippocampus at $72 \mathrm{~h}$ after SE (immunohistochemical analysis) Immunohistochemical images of caspase- 3 in hippocampal sections (the sham group, $n=5$; the other three groups, $n=5$ ). (A) Caspase- 3 was weakly expressed in the hippocampus of sham control rats. (B) Caspase-3 expression of the epilepsy group increased significantly in the hippocampal CA1 pyramidal cell layer at $72 \mathrm{~h}$. (C) Caspase-3 expression of the epilepsy plus saline group showed a similar pattern as that of the epilepsy group. (D) Simvastatin (1 mg/kg) abolished the increase in caspase-3 expression under SE treatment. (E) Number of caspase-3-positive neurons. " $\mathrm{P}<0.01$ vs. sham; ${ }^{\prime \prime} \mathrm{P}<0.01$ vs. epilepsy. Scale bars: lower magnification, $50 \mu \mathrm{m}$; higher magnification, $500 \mu \mathrm{m}$. so, stratum oriens; sp, stratum pyramidal; sr, stratum radiatum.

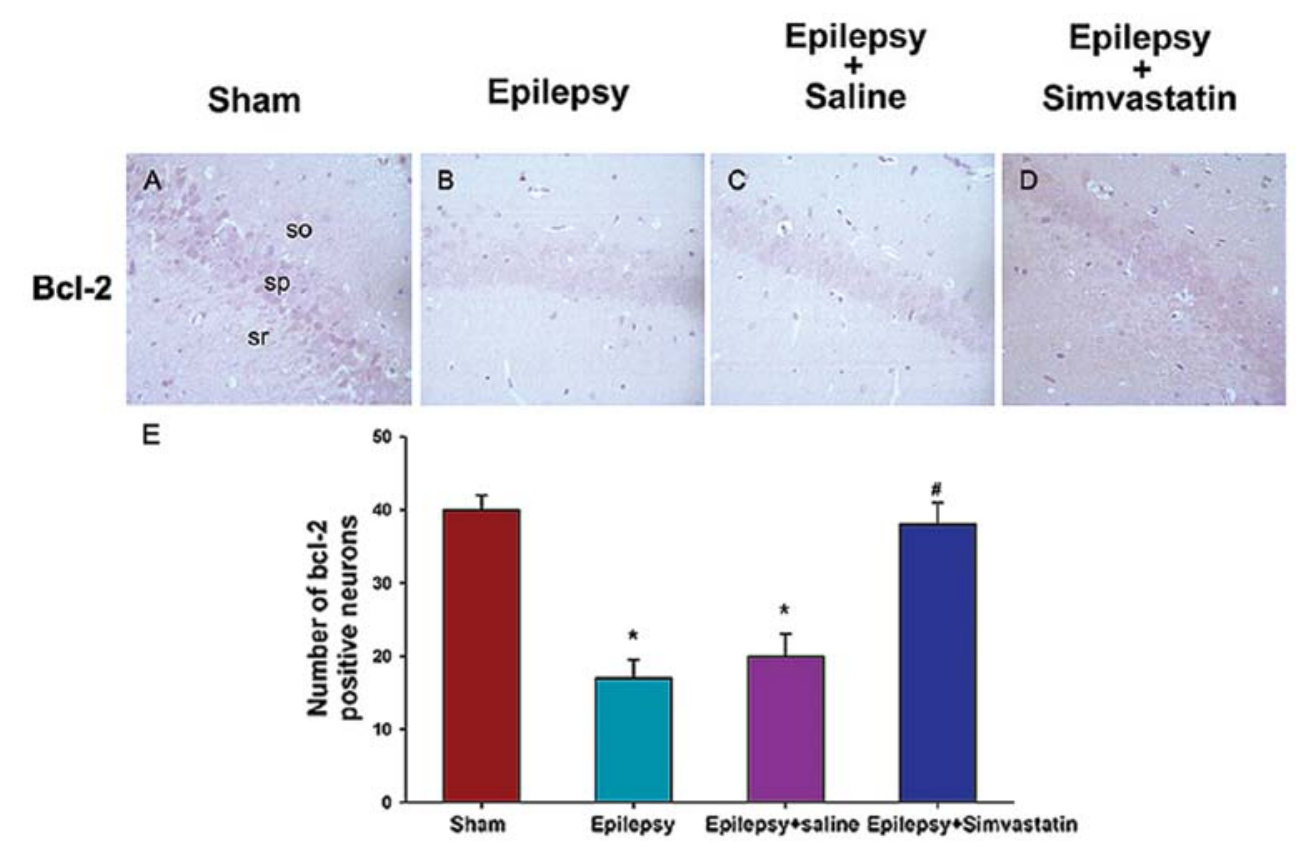

Figure 5. Simvastatin reverses SE-induced reduction in Bcl-2 protein expression in the hippocampus at $12 \mathrm{~h}$ after SE (immunohistochemical analysis). Immunohistochemical images of Bcl-2 in hippocampal sections (the sham group, $\mathrm{n}=5$; the other three groups, $\mathrm{n}=5$ ). (A) Bcl-2 staining in the hippocampus of sham control rats. (B) SE treatment significantly reduced Bcl-2 expression of the epilepsy group in the hippocampal CA1 pyramidal cell layer. (C) Bcl-2 expression of the epilepsy plus saline group showed a similar pattern as that of the epilepsy group. (D) Simvastatin (1 $\mathrm{mg} / \mathrm{kg}$ ) reversed SE-induced reduction in Bcl-2 expression. (E) Number of Bcl-2-positive neurons. "P<0.05 vs. sham; ${ }^{*} \mathrm{P}<0.05$ vs. epilepsy. Scale bars: lower magnification, $50 \mu \mathrm{m}$; higher magnification, $500 \mu \mathrm{m}$. so, stratum oriens; sp, stratum pyramidal; sr, stratum radiatum.

apoptosis by inhibiting Bcl-2 expression. Indeed, Bcl-2 overexpression provides modest protection against hippocampal seizure damage (38), whereas the level of SE-induced hippocampal neuronal death in Bcl-w-deficient mice is twice as severe as that in wild-type mice (39).
Statins are important drugs to ameliorate neurodegenerative diseases due to its significant neuroprotective roles as well as the fact that they are well tolerated with relatively few side effects. Recent clinical trials have reported the neuroprotective function of statins in Alzheimer's disease (40), Parkinson's 
disease $(41,42)$, and multiple sclerosis $(43)$. Although the exact mechanisms still remain unclear, several important discoveries have been made. First, statins reduce the circulating cholesterol levels and thus deplete lipid rafts, which contain many proteins involved in neurotransmitter systems (44) and have been linked to the processing of prion protein and the impairment of dopamine function in Parkinson's disease (45). Second, statins also exert cholesterol-independent effects by blocking the production of the non-sterol isoprenoid products of cholesterol synthesis, such as farnesylpyrophosphate and geranylgeranylpyrophosphate, which are active players in protein modification (46). Third, statins can influence the outcome of neurological insults through peripheral actions, including reducing oxidative damage, improving vascular function, and modulating peripheral inflammatory responses. Fourth, statins activate several neuroprotective and antiapoptotic signaling pathways, including the brain-derived neurotrophic factor pathway, the protein kinase B (PKB/Akt) pathway, and the Ras-ERK signaling cascade (47-49). In this study, we demonstrated that treatment with simvastatin significantly attenuates SE-induced neuronal loss and apoptosis in the hippocampus. Simvastatin abolishes SE-induced increase in caspase- 3 mRNA expression $24 \mathrm{~h}$ after the 2-h SE. A persistent reduction in caspase- 3 protein expression was also observed. In contrast, simvastatin could significantly reverse SE-induced reduction in Bcl-2 mRNA and protein expression levels. In conclusion, our results suggest that the mitochondrial apoptotic pathways participate in the protective effect of simvastatin against KA-evoked prolonged seizures.

Controversies over the clinical use of statins include several in vitro studies reporting that statins are actually neurotoxic and induce cell death in neurons and glia $(50,51)$. However, these experiments were usually carried out with very high statin concentrations of $0.1,1$ or even $10 \mathrm{mM}$. Thus, their physiological significance is unclear considering that the maximum concentration of pravastatin is only $0.1 \mathrm{mM}$ in the serum of healthy subjects (52). Moreover, statins probably do not reach this concentration in the central nervous system (53). Different types of statins differ in their ability to attenuate KA-induced seizures. Simvastatin is one of the most effective statins against kainate-induced excitotoxicity (54). Further studies are required to examine whether simvastatin can influence apoptotic regulators other than caspase- 3 and Bcl-2 and whether the anti-apoptotic properties of simvastatin depend on the dosages administered.

In conclusion, our study demonstrated that SE induces apoptosis in the rat hippocampus by increasing the expression of the pro-apoptotic caspase- 3 and reducing the expression of the anti-apoptotic $\mathrm{Bcl}-2$, in a time-dependent manner. Treatment with simvastatin rescues SE-induced apoptosis by reversing SE-produced changes in the expression of caspase-3 and Bcl-2. This study helps to further our understanding of the mechanisms of neuronal loss and apoptosis as well as the protective effects of simvastatin against SE-caused brain injury.

\section{References}

1. DeGiorgio CM, Tomiyasu U, Gott PS and Treiman DM: Hippocampal pyramidal cell loss in human status epilepticus. Epilepsia 33: 23-27, 1992.
2. Fujikawa DG, Itabashi HH, Wu A and Shinmei SS: Status epilepticus-induced neuronal loss in humans without systemic complications or epilepsy. Epilepsia 41: 981-991, 2000.

3. Pitkanen A, Kharatishvili I, Karhunen H, et al: Epileptogenesis in experimental models. Epilepsia 48 (Suppl 2): S13-S20, 2007.

4. Fujikawa DG, Shinmei SS and Cai B: Seizure-induced neurona necrosis: implications for programmed cell death mechanisms. Epilepsia 41 (Suppl 6): S9-S13, 2000.

5. Radley JJ and Jacobs BL: Pilocarpine-induced status epilepticus increases cell proliferation in the dentate gyrus of adult rats via a 5-HT1A receptor-dependent mechanism. Brain Res 966: 1-12, 2003.

6. Tokuhara D, Sakuma S, Hattori H, Matsuoka O and Yamano T: Kainic acid dose affects delayed cell death mechanism after status epilepticus. Brain Dev 29: 2-8, 2007.

7. Niquet J, Auvin S, Archie M, et al: Status epilepticus triggers caspase- 3 activation and necrosis in the immature rat brain. Epilepsia 48: 1203-1206, 2007.

8. Ben-Ari Y and Cossart R: Kainate, a double agent that generates seizures: two decades of progress. Trends Neurosci 23: 580-587, 2000.

9. Leite JP, Garcia-Cairasco N and Cavalheiro EA: New insights from the use of pilocarpine and kainate models. Epilepsy Res 50: 93-103, 2002.

10. Kovacs R, Schuchmann S, Gabriel S, Kann O, Kardos J and Heinemann U: Free radical-mediated cell damage after experimental status epilepticus in hippocampal slice cultures. J Neurophysiol 88: 2909-2918, 2002

11. Narkilahti S, Pirttila TJ, Lukasiuk K, Tuunanen J and Pitkanen A: Expression and activation of caspase 3 following status epilepticus in the rat. Eur J Neurosci 18: 1486-1496, 2003.

12. Ekdahl CT, Mohapel P, Elmer E and Lindvall O: Caspase inhibitors increase short-term survival of progenitor-cell progeny in the adult rat dentate gyrus following status epilepticus. Eur J Neurosci 14: 937-945, 2001.

13. Engel T, Murphy BM, Hatazaki S, et al: Reduced hippocampal damage and epileptic seizures after status epilepticus in mice lacking proapoptotic Puma. FASEB J 24: 853-861, 2010.

14. Pahan K, Sheikh FG, Namboodiri AM and Singh I: Lovastatin and phenylacetate inhibit the induction of nitric oxide synthase and cytokines in rat primary astrocytes, microglia, and macrophages. J Clin Invest 100: 2671-2679, 1997.

15. Balduini W, Mazzoni E, Carloni S, et al: Prophylactic but not delayed administration of simvastatin protects against long-lasting cognitive and morphological consequences of neonatal hypoxicischemic brain injury, reduces interleukin-1beta and tumor necrosis factor-alpha mRNA induction, and does not affect endothelial nitric oxide synthase expression. Stroke 34: 2007-2012, 2003.

16. Chen SF, Hung TH, Chen CC, et al: Lovastatin improves histological and functional outcomes and reduces inflammation after experimental traumatic brain injury. Life Sci 81: 288-298, 2007.

17. Endres M, Laufs U, Huang Z, et al: Stroke protection by 3-hydroxy-3-methylglutaryl (HMG)-CoA reductase inhibitors mediated by endothelial nitric oxide synthase. Proc Natl Acad Sci USA 95: 8880-8885, 1998.

18. Li G, Shofer JB, Rhew IC, et al: Age-varying association between statin use and incident Alzheimer's disease. J Am Geriatr Soc 58: 1311-1317, 2010.

19. Lu D, Goussev A, Chen J, et al: Atorvastatin reduces neurological deficit and increases synaptogenesis, angiogenesis, and neuronal survival in rats subjected to traumatic brain injury. J Neurotrauma 21: 21-32, 2004.

20. Wolozin B, Manger J, Bryant R, Cordy J, Green RC and McKee A: Re-assessing the relationship between cholesterol, statins and Alzheimer's disease. Acta Neurol Scand Suppl 185: 63-70, 2006.

21. Lee JK, Won JS, Singh AK and Singh I: Statin inhibits kainic acid-induced seizure and associated inflammation and hippocampal cell death. Neurosci Lett 440: 260-264, 2008.

22. Mahmood A, Goussev A, Kazmi H, Qu C, Lu D and Chopp M: Long-term benefits after treatment of traumatic brain injury with simvastatin in rats. Neurosurgery 65: 187-192, 2009.

23. Xie C, Sun J, Qiao W, et al: Administration of simvastatin after kainic acid-induced status epilepticus restrains chronic temporal lobe epilepsy. PLoS One 6: e24966, 2011.

24. Racine RJ: Modification of seizure activity by electrical stimulation. II. Motor seizure. Electroencephalogr Clin Neurophysiol 32: 281-294, 1972.

25. Bengzon J, Mohapel P, Ekdahl CT and Lindvall O: Neuronal apoptosis after brief and prolonged seizures. Prog Brain Res 135: 111-119, 2002. 
26. Collins JA, Schandi CA, Young KK, Vesely J and Willingham MC: Major DNA fragmentation is a late event in apoptosis. J Histochem Cytochem 45: 923-934, 1997.

27. Weiss S, Cataltepe O and Cole AJ: Anatomical studies of DNA fragmentation in rat brain after systemic kainate administration. Neuroscience 74: 541-551, 1996.

28. Bengzon J, Kokaia Z, Elmer E, Nanobashvili A, Kokaia M and Lindvall O: Apoptosis and proliferation of dentate gyrus neurons after single and intermittent limbic seizures. Proc Natl Acad Sci USA 94: 10432-10437, 1997.

29. Faherty CJ, Xanthoudakis S and Smeyne RJ: Caspase-3dependent neuronal death in the hippocampus following kainic acid treatment. Brain Res Mol Brain Res 70: 159-163, 1999.

30. Henshall DC, Clark RS, Adelson PD, Chen M, Watkins SC and Simon RP: Alterations in bcl-2 and caspase gene family protein expression in human temporal lobe epilepsy. Neurology 55: 250-257, 2000

31. Earnshaw WC, Martins LM and Kaufmann SH: Mammalian caspases: structure, activation, substrates, and functions during apoptosis. Annu Rev Biochem 68: 383-424, 1999.

32. Henshall DC, Chen J and Simon RP: Involvement of caspase-3like protease in the mechanism of cell death following focally evoked limbic seizures. J Neurochem 74: 1215-1223, 2000.

33. Kondratyev A and Gale K: Intracerebral injection of caspase-3 inhibitor prevents neuronal apoptosis after kainic acid-evoked status epilepticus. Brain Res Mol Brain Res 75: 216-224, 2000.

34. Ananth C, Thameem Dheen S, Gopalakrishnakone P and Kaur C: Domoic acid-induced neuronal damage in the rat hippocampus: changes in apoptosis related genes (bcl-2, bax, caspase-3) and microglial response. J Neurosci Res 66: 177-190, 2001.

35. Fujikawa DG, Ke X, Trinidad RB, Shinmei SS and Wu A: Caspase-3 is not activated in seizure-induced neuronal necrosis with internucleosomal DNA cleavage. J Neurochem 83: 229-240, 2002.

36. Dumas TC, McLaughlin JR, Ho DY, Lawrence MS and Sapolsky RM: Gene therapies that enhance hippocampal neuron survival after an excitotoxic insult are not equivalent in their ability to maintain synaptic transmission. Exp Neurol 166: 180-189, 2000.

37. Gross A, McDonnell JM and Korsmeyer SJ: BCL-2 family members and the mitochondria in apoptosis. Genes Dev 13: 1899-1911, 1999.

38. Phillips RG, Lawrence MS,HoDY and Sapolsky RM: Limitations in the neuroprotective potential of gene therapy with Bcl-2. Brain Res 859: 202-206, 2000.

39. Murphy B, Dunleavy M, Shinoda S, et al: Bcl-w protects hippocampus during experimental status epilepticus. Am J Pathol 171: $1258-1268,2007$.
40. Hoglund $\mathrm{K}$ and Blennow K: Effect of HMG-CoA reductase inhibitors on beta-amyloid peptide levels: implications for Alzheimer's disease. CNS Drugs 21: 449-462, 2007.

41. Huang X, Chen H, Miller WC, et al: Lower low-density lipoprotein cholesterol levels are associated with Parkinson's disease. Mov Disord 22: 377-381, 2007.

42. Wahner AD, Bronstein JM, Bordelon YM and Ritz B: Statin use and the risk of Parkinson disease. Neurology 70: 1418-1422, 2008.

43. Aktas O, Waiczies S, Smorodchenko A, et al: Treatment of relapsing paralysis in experimental encephalomyelitis by targeting Th1 cells through atorvastatin. J Exp Med 197: 725-733, 2003.

44. Allen JA, Halverson-Tamboli RA and Rasenick MM: Lipid raft microdomains and neurotransmitter signalling. Nat Rev Neurosci 8: 128-140, 2007.

45. Benarroch EE: Lipid rafts, protein scaffolds, and neurologic disease. Neurology 69: 1635-1639, 2007.

46. Greenwood J, Steinman L and Zamvil SS: Statin therapy and autoimmune disease: from protein prenylation to immunomodulation. Nat Rev Immunol 6: 358-370, 2006.

47. $\mathrm{Wu} \mathrm{H}$, Lu D, Jiang H, et al: Simvastatin-mediated upregulation of VEGF and BDNF, activation of the PI3K/Akt pathway, and increase of neurogenesis are associated with therapeutic improvement after traumatic brain injury. J Neurotrauma 25: 130-139, 2008

48. Dolga AM, Granic I, Nijholt IM, et al: Pretreatment with lovastatin prevents $\mathrm{N}$-methyl-D-aspartate-induced neurodegeneration in the magnocellular nucleus basalis and behavioral dysfunction. J Alzheimers Dis 17: 327-336, 2009.

49. Zipp F, Waiczies S, Aktas O, et al: Impact of HMG-CoA reductase inhibition on brain pathology. Trends Pharmacol Sci 28: 342-349, 2007

50. Marz P, Otten U and Miserez AR: Statins induce differentiation and cell death in neurons and astroglia. Glia 55: 1-12, 2007.

51. Xiang Z and Reeves SA: Simvastatin induces cell death in a mouse cerebellar slice culture (CSC) model of developmental myelination. Exp Neurol 215: 41-47, 2009.

52. Pan HY, Waclawski AP, Funke PT and Whigan D Pharmacokinetics of pravastatin in elderly versus young men and women. Ann Pharmacother 27: 1029-1033, 1993.

53. Botti RE, Triscari J, Pan HY and Zayat J: Concentrations of pravastatin and lovastatin in cerebrospinal fluid in healthy subjects. Clin Neuropharmacol 14: 256-261, 1991.

54. Ramirez C, Tercero I, Pineda A and Burgos JS: Simvastatin is the statin that most efficiently protects against kainate-induced excitotoxicity and memory impairment. J Alzheimers Dis 24: 161-174, 2011. 